\title{
Generalized Parton Distributions and Distribution of Partons in the Transverse Plane
}

\author{
M. Burkardt \\ Department of Physics, New Mexico State University, Las Cruces, NM 88011, USA \\ E-mail: burkardt@nmsu.edu
}

\begin{abstract}
I discuss the physical interpretation of GPDs in the limit $\xi=0$, where the $t$ dependence contains information about the distribution of partons in the transverse plane. GPDs thus allow a simultaneous determination of the longitudinal momentum and transverse position of partons in the infinite momentum frame, which also provides a physical interpretation for Ji's angular momentum sum rule.
\end{abstract}

\section{Motivation}

Recently, there has been a lot of interest in Generalized parton distributions (GPDs) because on the one hand GPDs can be probed in real and deeply virtual Compton scattering (DVCS) and on the other hand they can be related to the total angular momentum (including the orbital part!) carried by the quarks 1

$$
\text { DVCS } \Leftrightarrow \text { GPDs } \Leftrightarrow \vec{J}_{q} \text {. }
$$

However, even though GPDs clearly are interesting observables, it is not a priory clear whether they have a simple physical interpretation $\mathrm{B}$. In these notes, we will investigate the physics of GPDs in the limit where the momentum transfer on the target is purely transverse and we will show that the $t$-dependence of GPDs in this limit can be identified with the Fourier transform of parton distributions in the transverse plane w.r.t. the impact parameter.

\section{Generalized parton distributions}

GPDs are defined very similar to regular parton distributions

$$
\begin{aligned}
F_{q}(x, \xi, t) & =\int \frac{d x^{-}}{4 \pi} e^{i p^{+} x^{-} x}\left\langle P^{\prime}\left|\bar{q}\left(-\frac{x^{-}}{2}\right) \gamma^{+} q\left(\frac{x^{-}}{2}\right)\right| P\right\rangle \\
& =H_{q}(x, \xi, t) \frac{1}{2 p^{+}} \bar{u}\left(P^{\prime}\right) \gamma^{+} u(P)+E_{q}(x, \xi, t) \frac{1}{2 p^{+}} \bar{u}\left(P^{\prime}\right) \frac{i \sigma^{+\nu} \Delta_{\nu}}{2 M} u(P)
\end{aligned}
$$

where light front (LF) coordinates are defined as $x^{ \pm}=\frac{1}{\sqrt{2}}\left(x^{0} \pm x^{3}\right), \mathbf{x}_{\perp}=$ $\left(x^{1}, x^{2}\right)$, and $\Delta=p^{\prime}-p$. 
Unlike the familiar (forward) parton distribution functions (PDFs)

$$
q(x)=\int \frac{d x^{-}}{4 \pi} e^{i p^{+} x^{-} x}\left\langle P\left|\bar{q}\left(-\frac{x^{-}}{2}\right) \gamma^{+} q\left(\frac{x^{-}}{2}\right)\right| P\right\rangle,
$$

which have the physical meaning of a momentum density in the infinite momentum frame (IMF), GPDs do not have the meaning of a density but that of a probability amplitude:, GPDs describe the amplitude for finding a quark with momentum fraction $x-\xi$ (in the IMF) in the nucleon and replacing it back into the nucleon with a momentum transfer $\Delta^{\mu}$.

Several limiting cases are familiar: for $p^{\prime}=p$ one recovers the usual PDFs

$$
H(x, 0,0)=q(x) \quad \tilde{H}(x, 0,0)=\Delta q(x),
$$

while integration over $x$ yields the familiar form factors, e.g.

$$
\int d x H(x, \xi, t)=F_{1}(t) \quad \int d x E(x, \xi, t)=F_{2}(t) .
$$

Therefore another simple physical interpretation is that GPDs 'measure' the contribution of quarks with longitudinal momentum fraction $x$ to the corresponding form factor, i.e. GPDs are the form factor for the operator that 'filters out' quarks with longitudinal momentum fraction $x$ in the proton (in conventional PDFs, the quark is inserted back into the nucleon without momentum transfer).

Given the latter analogy, and given the fact that one can associate the Fourier transform of form factors with charge distributions in position space, it is very tempting to expect that GPDs also contain some information about the distribution of partons in position space.

\section{The physical interpretation of $H(x, 0, t)$}

In this section we will focus on the helicity non-flip case (unpolarized) Furthermore, we consider a situation when the momentum transfer between the initial and final state in Eq. (2) is purely transverse. In this limit, $F_{q}(x, 0, t)=H(x, 0, t)$ can be wriften as overlap integrals between LF wave functions (Fock space amplitudes $\Psi_{N}\left(x, \mathbf{k}_{\perp}\right)$

$$
H\left(x,-\boldsymbol{\Delta}_{\perp}^{2}\right)=\sum_{N} \sum_{j} \int[d x]_{N} \int\left[d^{2} \mathbf{k}_{\perp}\right]_{N} \delta\left(x-x_{j}\right) \Psi_{N}^{*}\left(x_{i}, \mathbf{k}_{\perp, i}^{\prime}\right) \Psi_{N}\left(x_{i}, \mathbf{k}_{\perp, i}\right)
$$

\footnotetext{
${ }^{a}$ Note that our results can be easily generalized to the polarized counterpart $\tilde{H}(x, 0, t)$.
} 
where $\mathbf{k}_{\perp, i}^{\prime}=\mathbf{k}_{\perp, i}-x_{i} \boldsymbol{\Delta}_{\perp}$ for $i \neq j$ and $\mathbf{k}_{\perp, j}^{\prime}=\mathbf{k}_{\perp, j}+\left(1-x_{j}\right) \boldsymbol{\Delta}_{\perp}$. Eq. (6) is very similar to the expression for the form factor in the Drell-Yan frame, except that the $x$ of the 'active' quark is not integrated over, and it is exact if one knows the $\Psi_{N}$ for all Fock components. The highly compact notation in Eq. (6) is illustrated by considering as an example the contributions from the two and three particle Fock components to $H\left(x, \mathbf{q}_{\perp}\right)$ for the pion

$$
\begin{aligned}
H\left(x,-\boldsymbol{\Delta}_{\perp}^{2}\right) & =\int d^{2} \mathbf{k}_{\perp} \psi_{\boldsymbol{\Delta}_{\perp}}^{*}\left(x, \mathbf{k}_{\perp}+\boldsymbol{\Delta}_{\perp}\right) \psi_{\mathbf{0}_{\perp}}\left(x, \mathbf{k}_{\perp}\right) \\
& +\int d^{2} \mathbf{k}_{\perp} d^{2} \mathbf{l}_{\perp} d y \psi_{\boldsymbol{\Delta}_{\perp}}^{*}\left(x, \mathbf{k}_{\perp}+\boldsymbol{\Delta}_{\perp}, y, \mathbf{k}_{\perp}\right) \psi_{\mathbf{0}_{\perp}}\left(x, \mathbf{k}_{\perp}, y, \mathbf{l}_{\perp}\right)+\ldots
\end{aligned}
$$

where '...' indicates contributions from 4 and more particle Fock components.

\subsection{Nonrelativistic form factors and charge density in position space}

Eq. (7) resembles expressions for the nonrelativistic (NR) form factor. For example for NR two \& three body systems, the form factor reads respectively

$$
\begin{aligned}
& F(\vec{q})=\int d^{3} \vec{k} \psi_{\vec{q}}^{*}(\vec{k}+\vec{q}) \psi_{\overrightarrow{0}}(\vec{k}) \\
& F(\vec{q})=\int d^{3} \vec{k}_{1} d^{3} \vec{k}_{2} \psi_{\vec{q}}^{*}\left(\vec{k}_{1}+\vec{q}, \vec{k}_{2}\right) \psi_{\overrightarrow{0}}\left(\vec{k}_{1}, \vec{k}_{2}\right) .
\end{aligned}
$$

Like GPDs, $F(\vec{q})$ is also off-diagonal in the momentum since the form factor is the non-forward $\left(\vec{P}^{\prime} \neq \vec{P}\right)$ matrix element of the vector current. In order to relate $F(\vec{q})$ to position space densities, one first uses that NR boosts are purely kinematic, i.e.

$$
\vec{k}_{i}^{\prime}=\vec{k}_{i}+x_{i} \vec{q}
$$

with $x_{i}=\frac{m_{i}}{M}$, to express the boosted wave functions in terms of wave functions in the rest frame

$$
\begin{aligned}
\psi_{\vec{q}}(\vec{k}) & =\psi_{\overrightarrow{0}}(\vec{k}-x \vec{q}) \\
\psi_{\vec{q}}\left(\vec{k}_{1}, \vec{k}_{2}\right) & =\psi_{\overrightarrow{0}}\left(\vec{k}_{1}-x_{1} \vec{q}, \vec{k}_{2}-x_{2} \vec{q}\right) .
\end{aligned}
$$

One can thus rewrite $F(\vec{q})$ as an autocorrelation of the $\vec{P}=\overrightarrow{0}$ wave function

$$
\begin{aligned}
& F(\vec{q})=\int d^{3} \vec{k} \psi_{\overrightarrow{0}}^{*}(\vec{k}+(1-x) \vec{q}) \psi_{\overrightarrow{0}}(\vec{k}) \\
& F(\vec{q})=\int d^{3} \vec{k}_{1} d^{3} \vec{k}_{2} \psi_{\overrightarrow{0}}^{*}\left(\vec{k}_{1}+\left(1-x_{1}\right) \vec{q}, \vec{k}_{2}-x_{2} \vec{q}\right) \psi_{\overrightarrow{0}}\left(\vec{k}_{1}, \vec{k}_{2}\right),
\end{aligned}
$$


for two and three particle systems respectively. Finally, upon Fourier transforming to position space, one can turn the autocorrelation function in Eq. (12) into a density. More precisely, one can express $F(\vec{q})$ in terms of the charge density $\rho(\vec{r})$ at distance $\vec{r}$ from the center of mass $\vec{R}_{C M} \equiv \sum_{i} \frac{m_{i}}{M} \vec{r}_{i}$.

$$
F(\vec{q})=\int d^{3} r e^{-i \vec{q} \cdot \vec{r}} \rho(\vec{r})
$$

\section{2 relevance for GPDs}

Purely transverse boosts in the LF frame also form a Galilean subgroup

$$
x_{i} \longrightarrow x_{i} \quad \mathbf{k}_{\perp, i} \longrightarrow \mathbf{k}_{\perp, i}+x_{i} \mathbf{q}_{\perp},
$$

where the momentum fractions $x_{i}$ play the role of the mass fractions $\frac{m_{i}}{M}$ in the NR case. Therefore, LF Fock space amplitudes transform under purely $\perp$ boosts very similar to the way NR wave functions transform. For example, for the two and three body Fock components one finds

$$
\begin{aligned}
\psi_{\mathbf{q}_{\perp}}\left(x, \mathbf{k}_{\perp}\right) & =\psi_{\mathbf{0}_{\perp}}\left(x, \mathbf{k}_{\perp}-x \mathbf{q}_{\perp}\right) \\
\psi_{\mathbf{q}_{\perp}}\left(x, \mathbf{k}_{\perp}, y, \mathbf{l}_{\perp}\right) & =\psi_{\mathbf{0}_{\perp}}\left(x, \mathbf{k}_{\perp}-x \mathbf{q}_{\perp}, y, \mathbf{l}_{\perp}-y \mathbf{q}_{\perp}\right) .
\end{aligned}
$$

Because of the very similar boost properties and the very similar expressions for GPDs one the one hand (7) and NR form factors on the other hand (8), one can proceed with GPDs in analogy with NR form factors to express them in terms of a density. First one expresses $H\left(x, \mathbf{q}_{\perp}\right)$ in terms of autocorrelations of Fock space amplitudes in the $\mathbf{p}_{\perp}=\mathbf{0}_{\perp}$ frame

$$
\begin{aligned}
& H\left(x,-\boldsymbol{\Delta}_{\perp}^{2}\right)=\int d^{2} \mathbf{k}_{\perp} \psi_{\mathbf{0}_{\perp}}^{*}\left(x, \mathbf{k}_{\perp}+(1-x) \boldsymbol{\Delta}_{\perp}\right) \psi_{\mathbf{0}_{\perp}}\left(x, \mathbf{k}_{\perp}\right) \\
& +\int d^{2} \mathbf{k}_{\perp} d^{2} \mathbf{l}_{\perp} d y \psi_{\mathbf{0}_{\perp}}^{*}\left(x, \mathbf{k}_{\perp}+(1-x) \boldsymbol{\Delta}_{\perp}, y, \mathbf{k}_{\perp}-x \boldsymbol{\Delta}_{\perp}\right) \psi_{\mathbf{0}_{\perp}}\left(x, \mathbf{k}_{\perp}, y, \mathbf{l}_{\perp}\right)+\ldots
\end{aligned}
$$

and then one Fourier transforms the $\perp$ coordinates to position space. In complete analogy with NR form factors this allows one to express $H(x, t)$ as the Fourier transform of parton distributions in the $\perp$ plane

$$
H\left(x,-\boldsymbol{\Delta}_{\perp}^{2}\right)=\int d^{2} \mathbf{b}_{\perp} e^{-i \boldsymbol{\Delta}_{\perp} \cdot \mathbf{b}_{\perp}} f\left(x, \mathbf{b}_{\perp}\right),
$$

where $f\left(x, \mathbf{b}_{\perp}\right)$ is the probability density to find a quark with momentum fraction $x$ at $\perp$ distance $\mathbf{b}_{\perp}$ from the $\perp$ center of momentum $\mathbf{R}_{\perp}^{C M} \equiv \sum_{i} x_{i} \mathbf{r}_{i, \perp}$. 
Note that $f\left(x, \mathbf{b}_{\perp}\right)$ is gauge invariant: to prove this, one uses that a formal operator definition of $f\left(x, \mathbf{b}_{\perp}\right)$, which in LF gauge is given by ${ }^{3}$

$$
f\left(x, \mathbf{b}_{\perp}\right)=\int \frac{d x^{-}}{4 \pi} e^{i p^{+} x^{-} x}\left\langle\psi_{l o c}\left|\bar{q}\left(-\frac{x^{-}}{2}, \mathbf{b}_{\perp}\right) \gamma^{+} q\left(\frac{x^{-}}{2}, \mathbf{b}_{\perp}\right)\right| \psi_{l o c}\right\rangle
$$

Here $\left|\psi_{l o c}\right\rangle \equiv \int d^{2} p_{\perp} \psi\left(\mathbf{p}_{\perp}\right)|p\rangle$ is a wave packet of plane wave proton states which is very localized in the transverse direction, but still has a sharp longitudinal momentum. This can be accomplished by taking $\psi\left(\mathbf{p}_{\perp}\right)=$ const., which guarantees that the $\perp$ center of momentum (the variable conjugate to the total $\perp$ momentum!) is at the origin. To make Eq. (18) manifestly gauge invariant one inserts a straight line gauge string from $\left(-\frac{x^{-}}{2}, \mathbf{b}_{\perp}\right)$ to $\left(\frac{x^{-}}{2}, \mathbf{b}_{\perp}\right)$.

This example also illustrates why the $\perp$ center of momentum is of significance here: first of all, the intrinsic dynamics of the hadron is independent of the overall $\perp$ momentum in the IMF ( $\perp$ boosts are purely kinematical), which allows to localize the the wave packet in the $\perp$ direction through a wave packet. Since the variable conjugate to the overall $\perp$ momentum is $\mathbf{R}_{\perp}^{C M}$, localizing the wave packet is equivalent to working in the frame where $\mathbf{R}_{\perp}^{\bar{C} M}=0$. P

\section{Discussion}

By means of a Fourier transform, $H\left(x,-\boldsymbol{\Delta}_{\perp}^{2}\right)$ tells us how partons are distributed in the transverse plane as a function of the distance from the $\perp$ center of momentum. First of all this provides us, at least for $\xi=0$, with a very simple interpretation for GPDs in terms of a density. A similar interpretation exists for $\tilde{H}\left(x,-\Delta_{\perp}^{2}\right)$. in terms of polarized impact parameter dependent quark distributions in a longitudinally polarized target. These fundamental results by themselves are already important observations because they illustrate the kind of physics that one can learn about hadrons by studying GPDs. However, above and beyond this physics insight, our results also have a number of practical application because they allow one to use geometric insights about hadron structure to model GPDs as illustrated by the following examples:

1. First of all, the mere fact that the $\mathbf{b}_{\perp}$ distribution is measured w.r.t. $\mathbf{R}_{\perp}^{C M}$ necessarily implies that the width of the $\mathbf{b}_{\perp}$ distribution goes to zero as $x \rightarrow 1$, since the active quark becomes the $\perp$ center of mass in that limit. In momentum space this implies that $H(x, t)$ should become $t$-independent as $x \rightarrow 1$. Many models for GPDs are consistent with this kind of behavior, but our analysis indicates that this is a general result.

${ }^{b}$ Of course the same reasoning also applies to the familiar case of the NR form factor. 
2. Eq. (18) illustrates that the impact parameter dependent parton distributions can be expressed in the form

$$
\left.f\left(x, \mathbf{b}_{\perp}\right) \sim\left\langle\psi_{l o c}\left|b^{\dagger}\left(x p^{+}, \mathbf{b}_{\perp}\right) b\left(x p^{+}, \mathbf{b}_{\perp}\right)\right| \psi_{l o c}\right\rangle=\left|b\left(x p^{+}, \mathbf{b}_{\perp}\right)\right| \psi_{l o c}\right\rangle\left.\right|^{2},
$$

where $b\left(x p^{+}, \mathbf{b}_{\perp}\right)$ creates quarks of long. momentum $x p^{+}$at $\perp$ position $\mathbf{b}_{\perp}$. This implies that $f\left(x, \mathbf{b}_{\perp}\right)$ has a probabilistic interpretation, and therefore it has to satisfy positivity constraints, i.e.

$$
f_{q}\left(x, \mathbf{b}_{\perp}\right) \equiv \int d^{2} \boldsymbol{\Delta}_{\perp} H_{q}\left(x, 0,-\boldsymbol{\Delta}_{\perp}^{2}\right) e^{i \boldsymbol{\Delta}_{\perp} \cdot \mathbf{b}_{\perp}}
$$

should be positive for all $x$ and all $\mathbf{b}_{\perp}$ (for antiquarks the same holds modulo an obvious sign). This positivity condition provides us with a new constraint on all models for GPDs.

3. Since very little is known about the actual $t$-dependence of GPDs, it is often convenient to use simple parameterizations. A commonly used form (motivated by LF constituent models) is $H\left(x, 0,-\boldsymbol{\Delta}_{\perp}^{2}\right)=q(x) e^{-a \Delta_{\perp}^{2} \frac{1-x}{x}}$. However, such a $\boldsymbol{\Delta}_{\perp}$-dependence yields an unacceptably rapid growth for the $\perp$ hadron size $\left\langle\mathbf{b}_{\perp}^{2}\right\rangle \sim \frac{1}{x}$ in the small $x$ region. Space time descriptions of parton structure in the small $x$ region suggest a $\perp$ size of hadrons that grows like $\alpha \ln \frac{1}{x}$. Translated into GPDs this implies that the small $x$ behavior should be parameterized by a functional form like

$$
H\left(x, 0,-\boldsymbol{\Delta}_{\perp}^{2}\right)=q(x) e^{-\alpha \Delta_{\perp}^{2} \ln \frac{1}{x}} \quad \text { or } \quad q(x) e^{-\alpha \boldsymbol{\Delta}_{\perp}^{2}(1-x) \ln \frac{1}{x}}
$$

(the $2^{\text {nd }}$ ansatz is also consistent with the Drell-Yan-West relation).

4. At a more qualitative level, one expects quarks at large $x$ to come from the more localized valence 'core' of the hadron, while the small $x$ region should also receive contributions from the much wider meson 'cloud' and therefore one would in general expect a gradual increase of the $t$ dependence of $H(x, 0, t)$ as one goes from larger to smaller values of $x$

\section{The Physics of $E(x, 0, t)$}

So far we have only considered GPDs in an unpolarized (or longitudinally) polarized nucleon. For polarized nucleon states (we use an IMF helicity basis here ) one finds the following useful relations when $\Delta^{+}=0$

$$
\begin{aligned}
& \int \frac{d x^{-}}{4 \pi} e^{i p^{+} x^{-} x}\left\langle P+\Delta, \uparrow\left|\bar{q}(0) \gamma^{+} q\left(x^{-}\right)\right| P, \uparrow\right\rangle=H\left(x, 0,-\Delta_{\perp}^{2}\right) \\
& \int \frac{d x^{-}}{4 \pi} e^{i p^{+} x^{-} x}\left\langle P+\Delta, \uparrow\left|\bar{q}(0) \gamma^{+} q\left(x^{-}\right)\right| P, \downarrow\right\rangle=-\frac{\Delta_{x}-i \Delta_{y}}{2 M} E\left(x, 0,-\Delta_{\perp}^{2}\right),
\end{aligned}
$$


i.e. if we take for example a nucleon polarized in the $x$ direction (in the IMF)

$$
F_{q}\left(x, 0,-\boldsymbol{\Delta}_{\perp}\right)=H\left(x, 0,-\boldsymbol{\Delta}_{\perp}^{2}\right)+i \frac{\Delta_{y}}{M} E\left(x, 0,-\boldsymbol{\Delta}_{\perp}^{2}\right) .
$$

Eq. (23) allows us to draw the following conclusions for the physics of $E(x, 0, t)$

1. We know already that $H\left(x, 0,-\boldsymbol{\Delta}_{\perp}^{2}\right)$ describes the distribution of unpolarized partons in the $\perp$ plane for a nucleon that is either unpolarized or polarized in the $z$ direction. From Eq. (23) we can immediately read off that $\frac{\Delta_{\perp}}{M} E\left(x, 0,-\boldsymbol{\Delta}_{\perp}^{2}\right)$ describes how the distribution of partons in the $\perp$ plane depends on the polarization of the nucleon. For example, for a nucleon polarized in the $x$ direction the z-momentum distribution of partons on the $+\mathrm{y}$ side will differ from the one on the $-y$ side - which makes sense if one considers the classical picture of a sphere that spins around the $\mathrm{x}$-direction while moving in the $\mathrm{z}$-direction. The z-momenta due to spinning add to the translatory momenta for 'partons' on the $+y$ side and subtract on the $-y$ side and hence it is not surprising to find a difference between the parton distributions on the $\pm y$ sides.

2. Since the probabilistic interpretation should not depend on the polarization, above interpretation provides us with another positivity constraint relating the Fourier transform of $E$ to the one of $H$ :

$$
\left|\frac{\nabla_{b_{\perp}}}{M} \int d^{2} \mathbf{b}_{\perp} e^{i \mathbf{b}_{\perp} \cdot \boldsymbol{\Delta}_{\perp}} E\left(x, 0,-\boldsymbol{\Delta}_{\perp}^{2}\right)\right|<\int d^{2} \mathbf{b}_{\perp} e^{i \mathbf{b}_{\perp} \cdot \boldsymbol{\Delta}_{\perp}} H\left(x, 0,-\boldsymbol{\Delta}_{\perp}^{2}\right) .
$$

3. Finally, we are also able to illustrate the physics of Ji's angular momentum sum rule $\left\langle J_{q}\right\rangle=\frac{1}{2} \int d x x\left[H_{q}(x, 0,0)+E_{q}(x, 0,0)\right]$. For this purpose, we first note that GPDs for $\xi=0$ allow the simultaneous determination of the momentum of partons in the $z$ direction and their position in the $\perp$ direction (which is very much reminiscent of angular momentum $\left.L_{x}=x p_{z}-z p_{x}\right)$ and it should thus not surprise that GPDs allow determining the total angular momentum of the quarks. In order to illustrate this connection, let us consider a nucleon polarized in the $x$ direction Because we are interested in the angular momentum in the rest frame of the target, we again need to consider again nucleons polarized in the

${ }^{c}$ I was only able to find a simple physical interpretation for the connection between GPDs and the $\perp$ components of $\vec{J}_{q}$. However, by rotational invariance, one can apply the result also to $J_{q}^{z}$, and, by boost invariance, $J_{q}^{z}$ should also be he same for a nucleon moving in the $z$-direction. 
$x$-direction, but now in the nucleon rest frame. This differs from the IMF by a Melosh rotation and one finds up to terms linear in $\Delta_{\perp}$

$$
F_{q}\left(x, 0, \boldsymbol{\Delta}_{\perp}\right)=H\left(x, 0,-\boldsymbol{\Delta}_{\perp}^{2}\right)+i \frac{\Delta_{y}}{2 M}\left[H\left(x, 0,-\boldsymbol{\Delta}_{\perp}^{2}\right)+E\left(x, 0,-\boldsymbol{\Delta}_{\perp}^{2}\right)\right] .
$$

For the $x$ component of the angular momentum carried by the quarks one thus finds ${ }^{a}$

$$
\begin{aligned}
\left\langle J_{q}^{x}\right\rangle & =\left\langle y T^{0 z}\right\rangle-\left\langle z T^{0 y}\right\rangle=2\left\langle y T^{0 z}\right\rangle=2 \frac{M}{2} \int d x \int d^{2} \mathbf{b}_{\perp} b_{y} x f\left(x, \mathbf{b}_{\perp}\right) \\
& =\left.M i \frac{d}{d \Delta_{y}} \int d x x F_{q}\left(x, 0, \boldsymbol{\Delta}_{\perp}\right)\right|_{\Delta=0}=\frac{1}{2} \int d x x[H(x, 0,0)+E(x, 0,0)]
\end{aligned}
$$

which is the angular momentum relation derived in Ref. 6 . What is new is that through the geometric interpretation of GPDs, we are now able to understand the physics of this sum rule by relating GPDs to the expectation value of $\left\langle y p_{z}\right\rangle$ for a nucleon polarized in the $x$ direction.

\section{Acknowledgments}

This work was supported by a grant from DOE (FG03-95ER40965) and through Jefferson Lab by contract DE-AC05-84ER40150 under which the Southeastern Universities Research Association (SURA) operates the Thomas Jefferson National Accelerator Facility.

1. X. Ji, Phys. Rev. Lett. 78, 610 (1997).

2. X. Ji in Future Directions in Quark Nuclear Physics, eds. A.W. Thomas and K. Tsushima (World Scientific, Singapore, 1999).

3. M. Burkardt, Phys. Rev. D 62, 071503 (2000).

4. M. Burkardt, hep-ph/0007036; hep-ph/0008051; hep-ph/0010082,

5. M. Diehl et al., Eur. Phys. Journal C 8, 409 (1999); Nucl. Phys. B 596, 33 (2001); B.C. Tiburzi and G.A. Miller, hep-ph/0104198.

6. V.N. Gribov, hep-ph/0006158.

7. S.J. Brodsky et al., Nucl. Phys. B 593, 311 (2001)

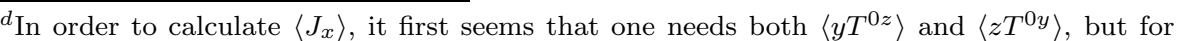
stationary localized states the two expectation values are (up to a sign) the same and it is thus sufficient to calculate $\left\langle y T^{0 z}\right\rangle$. Furthermore, in the rest frame one can replace the usual momentum density in $\left\langle y T^{0 z}\right\rangle$ by the LF momentum density $T^{++}$, whose matrix elements can be expressed by the GPDs.
} 